University of Wollongong

Research Online

Faculty of Informatics - Papers (Archive)

Faculty of Engineering and Information

Sciences

1987

\title{
Generalized Hadamard matrices and colourable designs in the construction of regular GDDs with two and three association classes
}

Jennifer Seberry

University of Wollongong, jennie@uow.edu.au

Follow this and additional works at: https://ro.uow.edu.au/infopapers

Part of the Physical Sciences and Mathematics Commons

\section{Recommended Citation}

Seberry, Jennifer: Generalized Hadamard matrices and colourable designs in the construction of regular GDDs with two and three association classes 1987.

https://ro.uow.edu.au/infopapers/1023

Research Online is the open access institutional repository for the University of Wollongong. For further information contact the UOW Library: research-pubs@uow.edu.au 


\title{
Generalized Hadamard matrices and colourable designs in the construction of regular GDDs with two and three association classes
}

\author{
Abstract \\ Generalized Hadamard matrices and colourable designs are used to construct many new group divisible \\ designs. \\ Disciplines \\ Physical Sciences and Mathematics \\ Publication Details \\ Seberry, J, Generalized Hadamard matrices and colourable designs in the construction of regular GDDs \\ with two and three association classes, Journal of Statistical Planning and Inference, 15, 1987, 237-246.
}




\title{
GENERALIZED HADAMARD MATRICES AND COLOURABLE DESIGNS IN THE CONSTRUCTION OF REGULAR GDDS WITH TWO AND THREE ASSOCIATION CLASSES
}

\author{
Jennifer SEBERRY* \\ Basser Department of Computer Science, University of Sydney, N.S.W. 2006, Australia \\ Received 3 October 1985; revised manuscript received 10 March 1986 \\ Recommended by R.G. Stanton
}

\begin{abstract}
Generalized Hadamard matrices and colourable designs are used to construct many new group divisible designs.

AMS Subject Classification: 62K10, 05B05, 05B20, 05B15, 05B30, 51E30.
\end{abstract}

Key words: Partially balance incomplete block designs; Group divisible designs; Balanced incomplete designs; Colourable designs; Generalized Hadamard matrices; Latin squares.

\section{Introduction and preliminaries}

Recently the $(0,1)$-matrices of order $q^{2}$ (sometimes called auxiliary matrices) used by a number of authors (Wallis (1971), Glynn (1978), Drake (1979), Jungnickel (1979), Mavron (1981) and Rajkundlia (1979)) in various guises, have been used in the construction of new families of Williamson type matrices (Seberry (1986)), the construction of new conference matrices (Seberry and Whiteman (1987)), the construction of new generalized Hadamard matrices (de Launey (1984)), and of new GDDs (Rodger, Sarvate and Seberry (1987)).

Auxiliary matrices, $B_{i}$, obey a number of equations including $B_{i} B_{j}^{\mathrm{T}}=J, i \neq j$. This paper studies and applies matrices, $A_{i}$, analogous to auxiliary matrices, which satisfy $A_{i} A_{j}^{\mathrm{T}}=h J, i \neq j, h>1$. These new matrices are obtained from generalized Hadamard matrices $\mathrm{GH}(h g, G),|G|=g, h>1$. Let $G$ be a finite group of order $g$. Let $e$ be the identity element of $G$ and let $R(G)$ denote the group ring of $G$. Let $J_{n, s}$ denote the $n \times s$ matrix whose entries are all 1 . When $s=n$ we abbreviate $J_{n, s}$ to $J_{n}$.

A generalized Hadamard matrix $\mathrm{GH}(g h, G)=\left(g_{i j}\right)=H$ over the group $G$ of order $g$ is a $g h \times g h$ matrix such that

(i) $g_{i j} \in G$ for all $1 \leq i, j \leq g h$, and

* Research supported by grants from ARGS and ACRB. 
(ii) $\sum_{k=1}^{g h} g_{i k} g_{j k}^{-1}=\sum_{a \in G} h a$ whenever $i \neq j$ where the summation is in the group ring $R(G)$. We also write this as

$$
H H^{\dagger}=h G \text {. }
$$

Several families of generalized Hadamard matrices have been found by Butson (1962,63), Drake (1979), Seberry (1978), Jungnickel (1979), Seiden (1954), Street (1979), Dawson (1985). Drake (1978) has also given various ways of combining known generalized Hadamard matrices to yield others. Non-existence of generalized Hadamard matrices has been explored by de Launey (1984).

Generalized Hadamard matrix existence theorem. Let $\mathrm{EA}\left(p^{i}\right)$ be the elementary abelian group of order $p^{i}$, where $p$ is prime. Then the following generalized Hadamard matrices exist:

(i) (a) $\mathrm{GH}\left(p^{i}, \mathrm{EA}\left(p^{i}\right)\right)$ for all $i \geq 1$;

(b) $\mathrm{GH}\left(p^{i+j}, \mathrm{EA}\left(p^{i}\right)\right)$ for all $i \geq 1, j \geq 0$;

(ii) $\mathrm{GH}\left(2^{m} p^{i}, \mathrm{EA}(p)\right)$ for all $0 \leq m \leq k, k \geq 1$;

(iii) (a) $\mathrm{GH}\left(2 p^{i}, \mathrm{EA}\left(p^{i}\right)\right)$ for all $i \geq 1$;

(b) $\mathrm{GH}\left(4 p^{i}, \mathrm{EA}\left(p^{i}\right)\right)$ for all $i \geq 1$;

(iv) if $p^{r}-1=q^{s}$ for some prime $q$ then there exist

(a) $\mathrm{GH}\left(p^{r}\left(p^{r}-1\right), \mathrm{EA}\left(p^{r}\right)\right)$ for all $r \geq 1$;

(b) $\mathrm{GH}\left(p^{r k+h}\left(p^{r}-1\right)^{j}, \mathrm{EA}\left(p^{i}\right)\right)$ for all $1 \leq i \leq r, 1 \leq j \leq k, h \geq 0$;

(c) $\mathrm{GH}\left(2^{m} p^{k+r i+h}\left(p^{r}-1\right)^{j}\right.$, $\left.\mathrm{EA}(p)\right)$ for all $0 \leq m \leq k, k \geq 1, i \geq j \geq 1, h \geq 0$.

Let $\mathrm{GH}(g h, G)=\left(g_{i j}\right)$ be a generalized Hadamard matrix with elements from the abelian group $G$ of order $|G|=g$. From $g h$ matrices $Y_{i}$ of order $g h$ by choosing

$$
Y_{i}=\left[\begin{array}{c}
g_{1 i} \\
g_{2 i} \\
\vdots \\
g_{g h, i}
\end{array}\right]\left(g_{1 i}, g_{2 i}, \ldots, g_{g h, i}\right) .
$$

Then over $R(g)$ the matrices $Y_{i}, i=1, \ldots, g h$, have the following properties (where $Y_{j}^{\dagger}$ means $Y^{\mathrm{T}}$ with the elements replaced by their inverses):

$$
\begin{aligned}
& Y_{i} Y_{j}^{\dagger}=h G \quad \text { whenever } i \neq j, \\
& \sum_{i=1}^{h g} Y_{i} Y_{i}^{\dagger}=h^{2} g G\left(J_{h g}-I_{h g}\right)+h^{2} g^{2} I_{h g} .
\end{aligned}
$$

Hence if we replace the entries of $Y_{i}$ by the usual permutation matrices we obtain $h g^{2} \times h g^{2}$ matrices, $A_{i}, i=1, \ldots, h g$, satisfying the following equations:

$$
\begin{aligned}
& A_{i} A_{j}^{\mathrm{T}}=h J_{h g^{2}}, \\
& A_{i} J_{h g^{2}}=h g J_{h g^{2}}, \\
& A_{i}\left(I_{h g} \times J_{g}\right)=J_{h g^{2}},
\end{aligned}
$$




$$
\sum_{i=1}^{h g} A_{i} A_{i}^{\mathrm{T}}=h^{2} g\left(\left(J_{h g}-I_{h g}\right) \times J_{g}\right)+h^{2} g^{2} I_{h g^{2}} .
$$

Example 1. $X=\mathrm{GH}\left(6, Z_{3}\right)$, writing the elements of $Z_{3}$ as $0,1,2$,

$$
X=\left(\begin{array}{llllll}
0 & 0 & 0 & 0 & 0 & 0 \\
0 & 0 & 1 & 2 & 2 & 1 \\
0 & 1 & 0 & 1 & 2 & 2 \\
0 & 2 & 1 & 0 & 1 & 2 \\
0 & 2 & 2 & 1 & 0 & 1 \\
0 & 1 & 2 & 2 & 1 & 0
\end{array}\right) .
$$

The 6 matrices of order $6, Y_{i}, i=1, \ldots, 6$, are

$$
\begin{aligned}
& \left(\begin{array}{llllll}
0 & 0 & 0 & 0 & 0 & 0 \\
0 & 0 & 0 & 0 & 0 & 0 \\
0 & 0 & 0 & 0 & 0 & 0 \\
0 & 0 & 0 & 0 & 0 & 0 \\
0 & 0 & 0 & 0 & 0 & 0 \\
0 & 0 & 0 & 0 & 0 & 0
\end{array}\right), \quad\left(\begin{array}{llllll}
0 & 0 & 1 & 2 & 2 & 1 \\
0 & 0 & 1 & 2 & 2 & 1 \\
1 & 1 & 2 & 0 & 0 & 2 \\
2 & 2 & 0 & 1 & 1 & 0 \\
2 & 2 & 0 & 1 & 1 & 0 \\
1 & 1 & 2 & 0 & 0 & 2
\end{array}\right), \quad\left[\begin{array}{llllll}
0 & 1 & 0 & 1 & 2 & 2 \\
1 & 2 & 1 & 2 & 0 & 0 \\
0 & 1 & 0 & 1 & 2 & 2 \\
1 & 2 & 1 & 2 & 0 & 0 \\
2 & 0 & 2 & 0 & 1 & 1 \\
2 & 0 & 2 & 0 & 1 & 1
\end{array}\right), \\
& \left(\begin{array}{llllll}
0 & 2 & 1 & 0 & 1 & 2 \\
2 & 1 & 0 & 2 & 0 & 1 \\
1 & 0 & 2 & 1 & 2 & 0 \\
0 & 2 & 1 & 0 & 1 & 2 \\
1 & 0 & 2 & 1 & 2 & 0 \\
2 & 1 & 0 & 2 & 0 & 1
\end{array}\right), \quad\left[\begin{array}{llllll}
0 & 2 & 2 & 1 & 0 & 1 \\
2 & 1 & 1 & 0 & 2 & 0 \\
2 & 1 & 1 & 0 & 2 & 0 \\
1 & 0 & 0 & 2 & 1 & 2 \\
0 & 2 & 2 & 1 & 0 & 1 \\
1 & 0 & 0 & 2 & 1 & 2
\end{array}\right], \quad\left[\begin{array}{llllll}
0 & 1 & 2 & 2 & 1 & 0 \\
1 & 2 & 0 & 0 & 2 & 1 \\
2 & 0 & 1 & 1 & 0 & 2 \\
2 & 0 & 1 & 1 & 0 & 2 \\
1 & 2 & 0 & 0 & 2 & 1 \\
0 & 1 & 2 & 2 & 1 & 0
\end{array}\right),
\end{aligned}
$$

which satisfy

$$
Y_{i} Y_{j}^{\dagger}=2 Z_{3}\left(J_{6}\right), \quad i \neq j
$$

(that is two copies of $Z_{3}$ as each element) and

$$
\sum_{i=1}^{6} Y_{i} Y_{i}^{\dagger}=36 I_{6}+12 Z_{3}\left(J_{6}-I_{6}\right)
$$

We now replace the elements of $Z_{3}$ by the corresponding $3 \times 3$ permutation matrices to get six matrices, $A_{1}, \ldots, A_{6}$, of order 18 which satisfy

$$
\begin{aligned}
& A_{i} A_{j}^{\mathrm{T}}=2 J_{18}, \quad \sum_{i=1}^{6} A_{i} A_{i}^{\mathrm{T}}=36 I_{18}+12\left(J_{6}-I_{6}\right) \times I_{3}, \\
& A_{i} J_{18}=6 J_{18}, \quad A_{i}\left(I_{6} \times J_{3}\right)=J_{18} .
\end{aligned}
$$

The block cyclic matrices with first rows
(i) $A_{1} A_{2} \cdots A_{g h}$,
(ii) $I \times J_{g} A_{1} A_{2} \cdots A_{g h}$,
(iii) $J_{g h} A_{1} A_{2} \cdots A_{g h}$,
(iv) $0_{g h} A_{1} A_{2} \cdots A_{g h}\left(0_{g h}\right.$ the zero matrix),

give the required incidence matrices for the next theorem: 
Theorem 1. Suppose there exists a generalized Hadamard matrix $\mathrm{GH}(\mathrm{gh}, G)$. Then there exist regular group divisible designs with parameters

(i) $v=b=g^{2} h^{2}, r=k=g^{2} h^{2}, \lambda_{1}=0, \lambda_{2}=g h^{2}, m=g, n=g^{2} h^{2}$,

(ii) $\dot{v}=b=g^{2} h(g h+1), r=k=g^{2} h^{2}+g, \lambda_{1}=g, \lambda_{2}=g h^{2}, \lambda_{3}=g h^{2}-h+2$,

(iii) $v=b=g^{2} h(g h+1), \quad r=k=g^{2} h(h+1), \quad \lambda_{1}=g^{2} h, \quad \lambda_{2}=g h(g+\lambda), \quad \lambda_{3}=$ $h(g h+2 h-1)$,

(iv) $v=b=g^{2} h(g h+1), r=k=g^{2} h^{2}, \lambda_{1}=0, \lambda_{2}=g h^{2}, \lambda_{3}=h(g h-1)$.

Example 1 (continued). Using six matrices $A_{1}, \ldots, A_{6}$ of order 18 with $g=3$ and $h=2$ in the theorem we obtain regular group divisible designs with parameters:

(i) $v=b=108, r=k=36, \lambda_{1}=0, \lambda_{2}=12, m=3, n=36$;

(ii) $v=b=126, r=k=39, \lambda_{1}=3, \lambda_{2}=12, \lambda_{3}=12$;

(iii) $v=b=126, r=k=54, \lambda_{1}=18, \lambda_{2}=30, \lambda_{3}=22$;

(iv) $v=b=126, r=k=36, \lambda_{1}=0, \lambda_{2}=12, \lambda_{3}=10$.

Some further examples are given in Table 1. All these designs appear to be new.

Table 1

\begin{tabular}{|c|c|c|c|c|c|c|c|c|c|c|}
\hline \multirow{2}{*}{$\begin{array}{l}\text { Generalized } \\
\text { Hadamard matrix }\end{array}$} & \multicolumn{10}{|c|}{ PBIBD } \\
\hline & $g$ & $h$ & $\begin{array}{l}\text { Association } \\
\text { classes }\end{array}$ & $v$ & $b$ & $r$ & $k$ & $\lambda_{1}$ & $\lambda_{2}$ & $\lambda_{3}$ \\
\hline $\mathrm{GH}\left(10, Z_{5}\right)$ & 5 & 2 & 2 & 500 & 500 & 100 & 100 & 0 & 20 & \\
\hline $\mathrm{GH}\left(10, Z_{5}\right)$ & 5 & 2 & 3 & 550 & 550 & 105 & 105 & 5 & 20 & 20 \\
\hline $\mathrm{GH}\left(12, Z_{3}\right)$ & 3 & 4 & 2 & 432 & 432 & 144 & 144 & 0 & 48 & \\
\hline$G H\left(12, Z_{3}\right)$ & 3 & 4 & 3 & 468 & 468 & 147 & 147 & 3 & 48 & 46 \\
\hline $\mathrm{GH}\left(12, Z_{2} \times Z_{2}\right)$ & 4 & 3 & 2 & 576 & 576 & 144 & 144 & 0 & 36 & \\
\hline $\mathrm{GH}\left(12, Z_{2} \times Z_{2}\right)$ & 4 & 3 & 3 & 624 & 624 & 148 & 148 & 4 & 36 & 35 \\
\hline $\mathrm{GH}\left(20, Z_{5}\right)$ & 5 & 4 & 2 & 2000 & 2000 & 400 & 400 & 0 & 80 & \\
\hline $\mathrm{GH}\left(20, Z_{5}\right)$ & 5 & 4 & 3 & 2100 & 2100 & 405 & 405 & 5 & 80 & 77 \\
\hline
\end{tabular}

Theorem 2. Let $C$ be the incidence matrix of $a$ BIBD $(g h, b, r, h, \lambda)$ where $\lambda=h(h-1) /(g-1)$ and $r=\lambda+h^{2}$. Further let $A_{1}, A_{2}, \ldots, A_{g h}$ of order $g^{2} h^{2}$ be the matrices constructed above from a $\mathrm{GH}(g h, G)$, where $|G|=g$. Then

$$
D=\left[C \times J_{g} A_{1} A_{2} \cdots A_{g h}\right]
$$

is the incidence matrix of $a \mathrm{BIBD}\left(g^{2} h, g r^{1}, g h\left(g^{2} h-1\right) /(g-1), g h, g h(g h-1) /(g-1)\right)$.

Proof. Follows from

$$
\begin{aligned}
& D D^{\mathrm{T}}=((r-\lambda) I+\lambda J) \times g J+h^{2} g^{2} I_{h g^{2}}+h^{2} g J_{h g^{2}}-h^{2} g I_{h g} \times J_{g} \\
& =h^{2} g^{2} I+g h(g h-1) /(g-1) J, \\
& J D=g h J \text {. }
\end{aligned}
$$


Example 2. See the designs in Table 2.

Table 2

\begin{tabular}{lll}
\hline GH $(g h, \mathrm{EA}(g))$ & $C=\mathrm{BIBD}(g h, b, r, h, \lambda)$ & Constructed BIBD $\left(g^{2} h, g r^{1}, r^{1}, g h, \lambda^{1}\right)$ \\
\hline GH(6, EA(3)) & $(6,15,5,2,1)$ & $(18,153,51,6,15)$ \\
GH(12, EA(3)) & $(12,66,22,4,6)$ & $(36,630,210,12,66)$ \\
GH(12, EA(4)) & $(12,44,11,3,2)$ & $(48,752,188,12,44)$ \\
GH(9, EA(3)) & $(9,36,12,3,3)$ & $(27,351,117,9,36)$ \\
\hline
\end{tabular}

\section{Constructions using colourable designs}

As in Rodger, Sarvate and Seberry (1987), a BIBD or PBIBD with $r$ applications is called colourable if one of a set of $r$ (latin) symbols can be associated with each 1 in the incidence matrix of the design so that each symbol occurs exactly once per row and no symbol occurs more than once per column.

Theorem 3. Suppose there exists $a \mathrm{GH}(g h, G)$ and $a$ BIBD $(v, b, g h, k, \lambda)$ which is colourable. Then there exists a

$$
\text { GDD }\left(g^{2} h v, g^{2} h b, g^{2} h^{2}, g h k, \lambda_{1}=0, \lambda_{2}=h^{2} g, \lambda_{3}=h \lambda\right) \text {. }
$$

Proof. Replace the latin symbols by $A_{l}, \ldots, A_{g h}$ of order $g^{2} h$ and the zeros by the zero matrix of order $g^{2} h$.

Example 3. To illustrate we use the $\mathrm{GH}\left(6, Z_{3}\right)$ and note that the BIBDs with parameters $(6,6,6),(7,6,5),(4,8,6,3,2),(7,14,6,3,2),(7,21,6,2,1),(11,6,3)$ and $(13,6,1)$ are all colourable. Thus we have the designs in Table 3 . All these PBIBD appear to be new.

Table 3

\begin{tabular}{|c|c|c|c|c|c|c|c|c|c|c|c|}
\hline \multicolumn{5}{|c|}{ BIBD parameters } & \multicolumn{7}{|c|}{ PBIBD parameters } \\
\hline$v$ & $b$ & $r$ & $k$ & $\lambda$ & $v$ & $b$ & $r$ & $k$ & $\lambda_{1}$ & $\lambda_{2}$ & $\lambda_{3}$ \\
\hline 4 & 8 & 6 & 3 & 2 & 72 & 144 & 36 & 18 & 0 & 12 & 4 \\
\hline 6 & 6 & 6 & 6 & 6 & 108 & 108 & 36 & 36 & 0 & 12 & 12 \\
\hline 7 & 7 & 6 & 6 & 5 & 126 & 126 & 36 & 36 & 0 & 12 & 10 \\
\hline 7 & 14 & 6 & $\mathbf{3}$ & 2 & 126 & 252 & 36 & 18 & 0 & 12 & 4 \\
\hline 7 & 21 & 6 & 2 & 1 & 126 & 378 & 36 & 12 & 0 & 12 & 2 \\
\hline 11 & 11 & 6 & 6 & 3 & 198 & 198 & 36 & 36 & 0 & 12 & 6 \\
\hline 31 & 31 & 6 & 6 & 1 & 558 & 558 & 36 & 36 & 0 & 12 & 2 \\
\hline
\end{tabular}




\section{Constructions using semi-colourable designs}

A design (BIBD or PBIBD) is called semi-colourable if latin symbols can be associated with the incidence matrix of the design so that one distinguished symbol $A$, say, occurs a fixed number of times $(>1)$ in each row and every other symbol occurs exactly once in each row while in each column the symbols are all different. In addition the replacement of the distinguished symbol by zero must leave a new colourable design.

Example 4. The designs in the following cases are obtained by cycling the first rows of the initial block or blocks under the given modulus.

(i) (Aabc), (Adef) $\bmod 4$;

(ii) (Aab), (Acd), (Aef) mod 3;

(iii) (Aab0c00), (Ade0f00) $\bmod 7$;

(iv) (Aa00b), (AOcd0) $\bmod 5$;

(v) (0Aa0b00), (0cAd00), (0ef0A00) $\bmod 7$.

Construction Lemma 1. A semi-colourable design on $(s+1) t$ symbols $A, a_{1}, \ldots, a_{s t}$, where the distinguished element occurs t times, can be formed by cycling the tinitial blocks,

$$
\left(A a_{1}, a_{2} \cdots a_{s}\right),\left(A a_{s+1} a_{s+2} \cdots a_{2 s}\right), \ldots,\left(A a_{s t-s+1} \cdots a_{s t}\right) \bmod s+1 .
$$

The first block design has parameters

$$
(s+1,(s+1) t,(s+1) t, s+1,(s+1) t) ;
$$

the second block design (with the distinguished element removed) has parameters

$$
(s+1,(s+1) t, s t, s,(s-1) t) \text {. }
$$

See Examples 4(i), (ii).

Construction Lemma 2. Let $D_{i}=\left\{d_{i 1}, \ldots, d_{i s}\right\}, i=1, \ldots, t$, be initial blocks $\bmod n$ of a design ( $n, t n, s t, s, \lambda)$ with the property that $d_{i j} \neq 0$ for any $i, j, d_{i j} \in D_{i} \rightarrow-d_{i j} \notin D_{i}$. Then a semi-colourable design on $(s+1) t$ symbols $A, a_{11}, \ldots, a_{s t}$, where the distinguished element occurs $t$ times can be formed by associating $A$ with the zero element of each initial block $D_{i}$ and $a_{i j}$ with $d_{i j}$.

Now cycle the blocks. The new design has parameters

$$
(n, t n,(s+1) t, s+1, \lambda+t) \text {. }
$$

See Example 4(iii).

Construction Lemma 3. Let $X+Y$ be the incidence matrix of a design, where $Y$ is also the incidence matrix of a design that is colourable and $X$ is a design where the 
intersections between rows of $X$ is zero then $A X+Y$ (coloured) is a semi-colourable design.

See Example 4(iv).

Construction Lemma 4. Let $Y$ be the incidence matrix of a design with parameters $(v, b, r, k, \lambda)$ which is colourable with the symbols $a_{1}, \ldots, a_{r}$ and in which each symbol also occurs once per column. Take r copies of Know coloured with the symbols $a_{i 1}, \ldots, a_{i r}, i=1, \ldots, r$. Replace $a_{i i}, i=1, \ldots, r$, by the distinguished symbol $A$ to obtain a semi-colourable design with parameters $\left(v, b r, r^{2}, k, r \lambda\right)$ with the underlying second design $(v, b r, r(r-1), k, \lambda(r-1))$.

See Example 4(v).

These lemmas give us the general results:

Theorem 4. Suppose there exists $a \mathrm{GH}(g h, G),|G|=g$, and $a \operatorname{BIBD}(v, b, g h+f, k, \lambda)$ which is semi-colourable (the distinguished element occurring $f$ times per row with underlying design having parameters $(u, b, g h, k-1, \mu)$. Then there exists a

$$
\begin{gathered}
\text { GDD }\left(g^{2} h v, g^{2} h b, g^{2} h^{2}+g f, g h(k-1)+g,\right. \\
\left.\lambda_{1}=f g, \lambda_{2}=h^{2} g, \lambda_{3}=\lambda+\mu(h-1)\right) .
\end{gathered}
$$

Proof. Replace the distinguished element by $I_{g h} \times J_{g}$ and the other latin symbols by $A_{1}, \ldots, A_{g h}$ of order $g^{2} h$. Replace the zero elements by the zero matrix of order $g^{2} h$.

Corollary 5. Suppose there exists a $\mathrm{GH}(g h, G),|G|=g$. Then there exists a

$$
\begin{gathered}
\mathrm{GGD}\left(g^{2} h(g h+1), g^{2} h(g h+1), g^{2} h^{2}+g, g^{2} h^{2}+g,\right. \\
\left.\lambda_{1}=g, \lambda_{2}=h^{2} g, \lambda_{3}=g h^{2}-h+2\right) .
\end{gathered}
$$

Proof. Use the cyclic design with first row

$$
I_{g h} \times J_{g} A_{1} A_{2} \cdots A_{g h} .
$$

Corollary 6. Suppose there exists a $\mathrm{GH}(g h, G),|G|=g$. Then there exist

(i) GDD $\left(2 g^{2} h, 2 g^{3} h^{2}, g h(g h+1), g(h+1), \lambda_{1}=g^{2} h, \lambda_{2}=h^{2} g, \lambda_{3}=2 g h\right)$;

(ii) GDD $\left(g^{2} h(g+1), g^{2} h^{2}(g+1), g h(g h+1), \lambda_{1}=g h, \lambda_{2}=h^{2} g, \lambda_{3}=h(g h-h+2)\right)$;

(iii) GDD $\left(g^{2} h(h+1), g^{3} h(h+1), g^{2}\left(h^{2}+1\right), \lambda_{1}=g^{2}, \lambda_{2}=h^{2} g, \lambda_{3}=g\left(h^{2}-h+2\right)\right)$.

Proof. For (i) use the BIBD $(2,2 g h, 2 g h, 2,2 g h)$ as

$$
\left[\begin{array}{l}
A A_{1} A A_{2} \cdots A A_{g h} \\
A_{1} A A_{2} A \cdots A_{g h} A
\end{array}\right] .
$$

For (ii) use the BIBD $(g+1, h(g+1), h(g+1), g+1, h(g+1))$ with blocks which cycle 
the first rows

$$
\left(A A_{1} \cdots A_{g}\right),\left(A A_{g+1} \cdots A_{2 g}\right), \ldots,\left(A A_{g h-g} \cdots A_{g h}\right) .
$$

For (iii) we use the BIBD $(h+1, g(h+1), g(h+1), h+1, g(h+1))$, with blocks which cycle the first rows

$$
\left(A A_{1} \cdots A_{h}\right),\left(A A_{h+1} \cdots A_{2 h}\right), \ldots,\left(A A_{g h-h} \cdots A_{g h}\right) .
$$

Example 5. See Table 4.

Table 4

\begin{tabular}{llll}
\hline $\begin{array}{l}\text { Semicolourable } \\
\text { design }\end{array}$ & $\begin{array}{l}\text { Underlying } \\
\text { colourable design }\end{array}$ & $\begin{array}{l}\text { GH } \\
\text { exist? }\end{array}$ & New desigl \\
\hline$(0, b, g h+f, k, \lambda)$ & $(v, b, g h, k-1, \mu)$ & $g, h$ & $\left(g^{2} h v, g^{2} h b, g^{2} h^{2}+g f, g h(k-1)+g, \lambda_{1}, \lambda_{2}, \lambda_{3}\right)$ \\
\hline$(7,7,7,7,7)$ & $(7,7,6,6,5)$ & 3,2 & $(126,126,39,3,12,12)$ \\
$(4,8,8,4,8)$ & $(4,8,6,3,2)$ & 3,2 & $(72,144,42,21,6,12,10)$ \\
$(3,9,9,3,9)$ & $(3,9,6,2,3)$ & 3,2 & $(54,162,45,15,9,12,12)$ \\
$(2,12,12,2,12)$ & $(2,12,6,1,0)$ & 3,2 & $(36,176,54,15,18,12,12)$ \\
$(7,14,8,4,4)$ & $(7,14,6,3,2)$ & 3,2 & $(126,252,42,21,6,12,6)$ \\
$(7,21,9,3,3)$ & $(7,21,6,2,1)$ & 3,2 & $(126,378,45,15,9,12,4)$ \\
$(2,24,24,2,24)$ & $(2,24,12,1,0)$ & 3,4 & $(72,864,180,15,36,48,24)$ \\
$(2,24,24,2,24)$ & $(2,24,12,1,0)$ & 4,3 & $(96,1152,192,16,48,36,24)$ \\
$(3,18,18,3,18)$ & $(3,18,12,2,6)$ & 3,4 & $(108,648,162,27,18,48,36)$ \\
$(3,18,18,3,18)$ & $(3,18,12,2,6)$ & 4,3 & $(144,864,168,28,24,36,30)$ \\
$(5,15,15,3,15)$ & $(5,15,12,4,9)$ & 3,4 & $(180,540,153,51,9,48,42)$ \\
$(5,15,15,3,15)$ & $(5,15,12,4,9)$ & 4,3 & $(240,720,156,52,28,12,36,33)$ \\
$(5,30,30,3,6)$ & $(5,30,12,2,3)$ & 3,4 & $(180,1080,198,27,54,48,15)$ \\
$(5,30,30,3,6)$ & $(5,30,12,2,3)$ & 4,3 & $(240,1140,216,28,72,36,12)$ \\
$(7,28,16,4,6)$ & $(7,28,12,3,3)$ & 3,4 & $(252,1008,156,39,12,48,15)$ \\
$(7,28,16,4,6)$ & $(7,28,12,3,3)$ & 4,3 & $(336,1344,160,40,16,36,12)$ \\
\hline
\end{tabular}

\section{New designs from old}

Most of our construction has involved constructions starting from a BIBD $(v, b, g h, k, \lambda)$ to get new designs with parameters

$$
V=g^{2} h v, B, R, K, \lambda_{1}, \lambda_{2}, \lambda_{3} \text {. }
$$

We now note that if a BIBD $\left(0, b^{1}, r^{1}, K / g, \lambda^{1}\right), C$ say, exists then

$$
E=I_{g h} \times J_{g} \times C
$$

gives a

$$
\operatorname{GDD}\left(g^{2} h v, B^{1}, R^{1}, K, \lambda_{1}=r^{1} g, \lambda_{2}=g, \lambda_{3}=0\right)
$$


with the same association classes as if it has essentially been constructed via a similar Kronecker product design. This design may sometimes be usefully combined with another design to reduce the number of different values of $\lambda_{i}$. For instance:

Construction Lemma 5. Suppose there exists a $\mathrm{GH}(g h, G),|G|=g$. Further suppose there exists a BIBD $\left(g^{2} h^{2}, B^{\prime}, R^{\prime}, g h^{2}, \lambda\right)=C$. Then with $F$ the $g \times f$ matrix of ones,

$$
[F \times C \mid \text { design in Theorem 1(i)] }
$$

is $a$

$$
\operatorname{GDD}\left(g^{3} h^{2}, g^{3} h^{2}+B^{\prime} f, R^{\prime} f+g^{2} h^{2}, g^{2} h^{2}, \lambda_{1}=R^{\prime} f, \lambda_{2}=\lambda f+g h^{2}\right) \text {. }
$$

Example 6. Let $g=3, h=2$ and $C=\operatorname{BIBD}(36,105,35,12,11)$. Then the lemma gives a

$$
\operatorname{GDD}\left(108,105 f+108,35 f+36,36, \lambda_{1}=35 f, \lambda_{2}=11 f+12\right)
$$

where $f \geq 1$ is integer.

Construction Lemma 6. Suppose there exists a generalized Hadamard matrix $\mathrm{GH}(g h, G),|G|=g$. Further suppose there exists a BIBD $(g h, b, r, h, \lambda)=C$. Then there exists a

$$
\operatorname{GDD}\left(v^{\prime}=g^{2} h, b^{\prime}, r g+g^{2} h^{2}, g h, \lambda_{1}=r g, \lambda_{2}=\lambda g+g h^{2}\right) .
$$

Proof. The incidence matrix is

$$
\left[C \times J_{g} A_{1} A_{2} \cdots A_{g h}\right] .
$$

Example 7. There is a $\operatorname{BIBD}(6,15,5,2,1)$ and a $\mathrm{GH}\left(6, Z_{3}\right)$. Hence there exists a BIBD $(18,153,51,6,15)$.

Corollary 7. Let $q$ be a prime power. Then there exists a $\mathrm{GH}(2 q, \mathrm{EA}(q))$. Further there exists a BIBD $(2 q, q(2 q-1), 2 q-1,2,1)$. Hence there exists $a$

$$
\operatorname{GDD}\left(2 q^{2}, 6 q^{3}-q^{2}, 6 q^{2}-q, 2 q, \lambda_{1}=q(2 q-1), \lambda_{2}=5 q\right) \text {. }
$$

\section{References}

Butson, A.T. (1962). Generalized Hadamard matrices. Proc. Amer. Math. Soc. 13, 894-898.

Butson, A.T. (1963). Relations among generalized Hadamard matrices, relative difference sets, and maximal length recurring sequences. Canad. J. Math. 15, 42-48.

Dawson, J.E. (1985). A construction for generalized Hadamard matrices GH(4q, EA(q)). J. Statist. Plann. Inference 11, 103-110.

de Launey, W. (1984). On the non-existence of generalised Hadamard matrices. J. Statist. Plann. Inference 10, 385-396.

Drake, D.A. (1979). Partial $\lambda$-geometries and generalized matrices over groups. Canad. J. Math. 31, $617-627$. 
Geramita, A.V. and J. Seberry (1979). Orthogonal Designs: Quadratic Forms and Hadamard Matrices. Marcel Dekker, New York-Basel.

Glynn, D.G. (1978). Finite Projective Planes and Related Combinatorial Systems. Ph.D. Thesis, University of Adelaide.

Jungnickel, D.J. (1979). On difference families, resolvable transversal designs and generalized Hadamard matrices. Math. Z. 167, 49-60.

Mathon, R. (1978). Symmetric conference matrices of order $p q^{2}+1$. Canad. J. Math. 30, 321-331.

Mavron, V.C. (1981). Constructions for resolvable and related designs. Aequationes Math. 23, $131-145$.

Rajkundlia, D. (1978). Some techniques for constructing new infinite families of balanced incomplete block designs. Ph.D. Dissertation, Queen's University, Kingston, Canada; cf. also. Discrete Math. 44 (1983), 61-96.

Raghavarao, D. (1971). Construction and Combinatorial Problems in Design of Experiments. Wiley, New York.

Rodger, C.A., G. Sarvate and J. Seberry (1987). Coloured designs, new group divisible designs and pairwise balanced designs. J. Statist. Plann. Inference 15 (1987), to appear.

Seberry, J. (1980). A construction for generalized Hadamard matrices. J. Statist. Plann. Inference 4, 365-368.

Seberry, J. (1986). A construction for Williamson type matrices. Graphs and Combinatorics 2, 81-87.

Seberry, J. and A.L. Whiteman (1987). New Hadamard matrices and conference matrices obtained via Mathon's construction. Combinatorica, submitted.

Seberry Wallis, J. (1972). Hadamard matrices, Part IV. Combinatorics: Room Squares, Sum Free Sets, Hadamard Matrices, Lecture Notes in Mathematics, Vol. 292, Springer, Berlin-New York.

Seiden, E. (1954). Construction of orthogonal arrays. Ann. Math. Statist. 25, 151-156.

Shrikhande, S.S. (1964). Generalized Hadamard matrices and orthogonal arrays of strength 2. Canad. J. Math. 16, 736-740.

Street, D.J. (1979). Generalized Hadamard matrices, orthogonal arrays and F-squares. Ars Combinat. $8,131-141$.

Wallis, J. (1971). Some results on configurations. J. Austral. Math. Soc. 12, 378-384. 